\title{
Hearing Loss and Sixth Cranial Nerve Paresis after COVID-19
}

\author{
Noel Lorenzo-Villalba, Léa Pierre, Javier Guerrero-Niño, Xavier Jannot, Emmanuel Andrès \\ Service de Médecine Interne, Diabète et Maladies Métaboliques, Hôpitaux Universitaires de Strasboug, France
}

Doi: 10.12890/2022_003221 - European Journal of Case Reports in Internal Medicine - ○ EFIM 2022

Received: 27/01/2022

Accepted: 01/02/2022

Published: 23/02/2022

How to cite this article: Lorenzo-Villalba N, Pierre L, Guerrero-Niño J, Jannot X, Andrès E. Hearing loss and sixth cranial nerve paresis after COVID-19. EJCRIM 2022;9: doi:10.12890/2022_003221.

Conflicts of Interests: The authors declare there are no competing interests.

This article is licensed under a Commons Attribution Non-Commercial 4.0 License

\section{ABSTRACT}

An 80-year-old patient was admitted to the internal medicine department for binocular diplopia and hearing loss with sudden onset. The patient had presented with SARS-CoV-2 infection 3 weeks previously and had been admitted to hospital. Complete work-up including autoimmunity, serum and LCR viral serology and MRI did not allow a diagnosis to be established. The hypothesis of a microvascular origin or the previous SARS-CoV-2 infection was considered. The latter was retained in light of the temporal relationship, the absence of other pathologies after exhaustive work-up, and the clinical evolution.

\section{LEARNING POINTS}

- A temporal relationship between SARS-CoV-2 infection and symptoms in the absence of other pathologies is important for diagnosis.

- Mid or long-term follow-up is necessary in patients with unexplained symptoms after SARS-CoV-2 infection.

\section{KEYWORDS}

Sixth nerve paresis, auditory acuity, MRI

\section{INTRODUCTION}

Several reports have emerged describing post-infection and long-term consequences of COVID-19 and its effects on multiple systems. However, the physiopathological mechanisms are not currently fully understood.

\section{CASE DESCRIPTION}

An 80-year-old patient was admitted to the internal medicine department for binocular diplopia and hearing loss with sudden onset. The patient had presented with SARS-CoV-2 infection 3 weeks earlier requiring hospitalization and had received treatment with dexamethasone and LMWH with disease resolution. His past medical history was also relevant for a low-grade lymphoma under surveillance, monoclonal gammopathy of undetermined significance (MGUS), Lyme disease treated in 2005, hypertension and dyslipidaemia. He was on treatment with clopidogrel $75 \mathrm{mg}$ daily, perindopril $4 \mathrm{mg}$ daily, atorvastatin $20 \mathrm{mg}$ per day and tamsulosin $0.4 \mathrm{mg}$ at night. He had no drug or food allergies and was a non-smoker.

On physical examination, blood pressure was $130 / 80 \mathrm{mmHg}$, heart rate was 78 beats $/ \mathrm{min}$, the patient was afebrile and oxygen saturation was $98 \%$ on room air. The neurological examination revealed a right sixth nerve paresis with convergence impairment and loss of auditory acuity. The rest of the cranial pairs were normal. There were no other motor or sensory deficits, and no cerebellar syndrome or extrapyramidal syndrome. Heart sounds were regular and lungs were clear.

Laboratory analysis revealed a normal white cell count, as well as normal liver, thyroid, coagulation and renal function tests. Glycated haemoglobin was 5.8\%. A nasopharyngeal swab for SARS-CoV-2 was negative. 
The results of a lumbar puncture showed: CSF appearance clear; protein (CSF) $0.39 \mathrm{~g} / \mathrm{l}$ (N: 0.15-0.45); albumin 196 mg/l (N: 130-350); CSF-lgG 30.3 mg/l (N: 10.0-34.0); albumin quotient (CSF/serum) 5.0×10-3 (N: <9.5×10-3) and Tibbling-Link Index (Q IgG/Q Alb) 0.47 (N: $<0.70$ ), while an oligoclonal band test showed a mirror profile with the presence of identical IgG oligoclonal bands in CSF and blood.

The head CT showed no evidence of recent haemorrhage or ischaemia.

Serology for HBV, HCV, HIV1/HIV2 and syphilis was negative. Serology for HSV1, HSV2, VZV, enterovirus and Lyme in the CSF was negative. Antinuclear, Anti-dsDNA antibodies, p-ANCA and c-ANCA were negative. C3, C4 and total complement were within the normal ranges. Vitamin B1, B6, B12 and folic acid levels were normal. Serum electrophoreses showed no changes regarding the IgG monoclonal peak. Lymphocyte phenotyping showed a B lympho-proliferation CD19+, CD20+, CD5-, monotypic kappa (medium), FMC7+, CD23-, CD22+ (strong), CD38-, CD43-, CD79b+, CD81+, slgG+, CD180+ (strong). Anti-RACH and anti-MUSK antibodies were negative

The ENT examination revealed bilateral hypoacusis more accentuated on the left and bilateral tinnitus, but no otorrhoea or otalgia. Otoscopy revealed an unremarkable eardrum.

Magnetic resonance imaging showed a minimal recent punctiform right temporo-occipital ischaemic lesion and vascular leukopathy. A second MRI (Fiesta) focused on cranial nerves was performed but found no evidence of neuritis or sixth cranial mass syndrome. In this setting a second lumbar puncture was performed, interleukin 6 and 10 were within the normal range and phenotyping was normal.

After discussion with a neurologist and ENT specialists, the origin of the patient's symptoms was thought to be microvascular or related to the previous SARS-CoV-2 infection considering the delay between infection and symptom onset; this hypothesis was confirmed during follow-up. The patient was put on corticoids $1 \mathrm{mg} / \mathrm{kg} /$ day in a tapering dose until withdrawal.

Nine months after follow-up, the patient had clinically improved, the right sixth nerve paresis had resolved, and auditory acuity had significantly improved.

\section{DISCUSSION}

We have presented a case of neurological manifestations 3 weeks after COVID-19. After complete work-up, two initial hypotheses were considered: microvascular involvement or previous COVID-19.

Three potential mechanisms by which SARS-CoV-2 can affect the central nervous system (CNS) have been suggested: transsynaptic spread of the virus, dissemination through the blood-brain barrier, and immune-mediated lesions ${ }^{[1-3]}$. In addition, vascular involvement could result in neurological manifestations in SARS-CoV-2-associated cerebrovascular disease. The infection and endothelial cell dysfunction lead to the release of tissue factors activating the thrombotic pathway ${ }^{[2]}$ and/or an inflammatory pathway provoking vasculitis or the destabilization of pre-existing atherosclerotic plaques ${ }^{[2]}$.

The neurological manifestations of SARS-CoV-2 have already been reported in the literature and include: meningitis, myelitis, encephalitis, CNS vasculitis, disseminated acute encephalomyelitis and post-infection acute disseminated encephalomyelitis/post-infectious brainstem encephalitis, Guillain-Barré syndrome and stroke ${ }^{[1-4]}$.

In the case presented, symptoms started 3 weeks after infection. Other authors have reported anterior ischaemic optic neuropathy 1 week after COVID-19 resolution ${ }^{[5]}$. The temporal relationship, the absence of other pathologies after exhaustive work-up, and the clinical evolution led us to suspect that the neurological symptoms in our patient were related to SARS-CoV-2 infection.

\section{CONCLUSIONS}

Post-SARS-CoV-2 infection symptoms are still not well understood due to the complex pathological mechanisms related to this infection.

\section{REFERENCES}

Silva FSCAD, Bucur A, Rosado SN, Balhana SDS, Meneses-Oliveira CM. Neurological dysfunction associated with Covid-19. Rev Bras Ter Intensiva 2021 Apr-Jun;33(2):325. Ellul MA, Benjamin L, Singh B, Lant S, Michael BD, Easton A, et al. Neurological associations of COVID-19. Lancet Neurol 2020;19(9):767-783.

Ahmed MU, Hanif M, Ali MJ, Haider MA, Kherani D, Memon GM, et al. Neurological manifestations of COVID-19 (SARS-CoV-2): a review. Front Neurol $2020 ; 11: 518$.

Zubair AS, McAlpine LS, Gardin T, Farhadian S, Kuruvilla DE, Spudich S. Neuropathogenesis and neurological manifestations of the coronavirus in the age of coronavirus disease 2019: a review. JAMA Neurol 2020;77(8):1018-1027.

5. Golabchi K, Rezaee A, Aghadoost D, Hashemipour M. Anterior ischemic optic neuropathy as a rare manifestation of COVID-19: a case report. Future Virol 2021 Nov:10.2217/ fvl-2021-0068. doi: 10.2217/fvl-2021-0068. 